\title{
Reliability Assessment of a Gas Generating Station in Ogun State, Nigeria
}

\section{OLASUNKANMI, OG; ALAO, PO; ONAIFO, F; 1*OSIFEKO, MO; SHOLABI; JO}

\author{
Department of Electrical and Electronic Engineering, ${ }^{1}$ Department of Computer Engineering, Olabisi Onabanjo University, Nigeria
}

*Corresponding Author Email: osifeko.martins@oouagoiwoye.edu.ng

\begin{abstract}
This study evaluates and presents the reliability and availability of a power plant, Akute Power Plant, Ogun State, Nigeria, during the period of January - December, 2015. Time series load data on each generating unit were collected from Akute Power Plant daily operational log book from January to December 2015. Outages were classified into types, frequency and durations. The operational data for the above period was collected and analyzed using Reliability methods. Reliability conditions are defined with the collected data and types of the failure faced by each generating units. The most important reliability indices like mean time to repair (MTTR), mean time to failure (MTTF), mean time between failures (MTBF), repair rate $(\mu)$, failure rate $(\lambda)$ were determined based on the loss of load approach. The reliabilities of each generating unit were evaluated on monthly basis for one year and the average availability of G1, G2, G3, and G4 were $0.9701,0.9726,0.9767$, and 0.9707 respectively, while the reliability indices for G1, G2, G3, and G4 were 0.9860 , $0.9880,0.9854$, and 0.9840 respectively. Suggestions were made to minimize system unavailability in order to improve the reliabilities of the network.
\end{abstract}

\section{DOI: https://dx.doi.org/10.4314/jasem.v22i6.27}

Copyright: Copyright (C) 2018 Olasunkanmi et al. This is an open access article distributed under the Creative Commons Attribution License (CCL), which permits unrestricted use, distribution, and reproduction in any medium, provided the original work is properly cited.

Dates: Received: 17 May 2018; Revised: 27 June: 2018; Accepted: 04 July 2018

Keyword: Reliability Methods, Mean Time To Repair, Mean Time Between Failure, Failure Rate.

Due to its suitability for peak load service, a gas turbine is used in several fields such as aviation, power generation, oil and gas industry, and marine propulsion. The working medium of a gas turbine power plant is a permanent gas. A conventional gas turbine power plant consists of turbines, a compressor mounted on the same shaft or coupled to the turbine, a combustor and auxiliaries such as lubricant pump and oil system (Mangey, 2017). The ability of a power system to consistently perform its intended function, on demand and without degradation or failure is known as reliability. A power system is said to perform satisfactorily if it does not fail during the time of service, however, it is normal for devices to undergo failures and repairs during their entire useful life. The recent growth in the economy has resulted in the increase in electricity demand causing a corresponding rise in capacity utilization of each generating unit. In effect, the frequency to break down the power units increases, which brings lots of economic lose to the society. (Sheng, 2017). In developing countries, power system planning has become a difficult, but important process of providing the necessary information needed in decision-making about energy investments (Abdullah, 2011). To assist in planning, reliability studies are conducted on a long-term basis. However, short-term evaluations are also useful for day to day operating decisions (Billinton and Allan, 1988).
Reliability study in a generation system is divided into adequacy and security. The system adequacy is associated with static conditions and do not include system disturbances while the system security on the other hand is related to the ability of the system to respond to disturbances which arise within the system. (Arya et al., 2012). The most widely used reliability indices in the generating system adequacy assessment are the Loss of Load Expectation (LOLE) which is the average number of days on which the daily or hourly peak load is expected to exceed the available generating capacity and Loss of Energy Expectation (LOEE) which is the expected energy that will not be supplied by the generating system due to those occasions when the load demand exceeds available generating capacity. (Okwe and Inyama, 2013). (Deepak et al., 2014) evaluated the reliability study of Sunkoshi Hydro Power Station, Nepal with an installed capacity of 10.05 Megawatts (MW). The operational data for 4 years was collected and analyzed using Markov model. Due to the unstable nature of electricity generation in the country and improve operational efficiency, this study evaluates the reliability of a gas Generating station in Ogun state, Nigeria.

\section{MATERIALS AND METHODS}


Study Area: The reliability study was carried out at Akute Power Plant (APL) situated at Akute Odo. Ogun State. APL is a gas generating station comprising of 4 generating unit. Each unit having the capacity of $3.0375 \mathrm{MW}$ and supplying $11 \mathrm{KV}$ to the outgoing feeders making a total of $12.15 \mathrm{MW}$. APL supplies power to 4 feeders (IJU Intake, IJU HEADWORKS , ADIYAN INTAKE , ADIYAN HEADWORKS feeder), with each end user units having their own loads in form of pumps, which pumps water from OGUN RIVER to their respective Head Works for further treatment before supplying to their respective customers. IJU INTAKE demands a total load of $(6 \times 500 \mathrm{KW}$ and $5 \times 300 \mathrm{KW}=4.5 \mathrm{MW})$, while its Head Work total load of $(5 \times 300 \mathrm{MW}=1.5 \mathrm{MW})$, also ADIYAN Intake demands a total load of $(5 \times 1.4 \mathrm{MW}=$ 7MW), while ADIYAN HEADWORKS demands a total load of $(7 \times 185 \mathrm{KW}=1.295 \mathrm{MW})$.

Data Collection: The outage data collected from Akute Power Plant comprises of information on each failure event within the period of January to December 2015. The information recorded in a narrative form was translated into a statistical database and is shown in Table 1.

Table 1. The Gas Plant Outage data for Jan - Dec 2015

\begin{tabular}{|c|c|c|c|c|c|c|}
\hline \multirow[t]{2}{*}{ Month(s) } & \multicolumn{2}{|c|}{ Forced Outage (FO) } & \multicolumn{2}{|c|}{ Scheduled Outage (SO) } & \multicolumn{2}{|c|}{ Total Ovtage (TO) } \\
\hline & Freq. & Duration [hr.] & Freq. & Duration [hr.] & Freq. & Duration [hr.] \\
\hline Jan & 3 & 0.52 & 3 & 32.25 & 6 & 32.77 \\
\hline Feb & 3 & 2.08 & 5 & 7.53 & 8 & 9.61 \\
\hline Mar & 4 & 0.83 & $=$ & - & 4 & 0.83 \\
\hline Apr & 5 & 0.95 & 2 & 12.00 & 7 & 12.95 \\
\hline May & 7 & 0.58 & $\overline{1}$ & 5.67 & 8 & 6.25 \\
\hline June & 7 & 1.82 & 1 & 7.50 & 8 & 9.32 \\
\hline July & 4 & 1.68 & 2 & 9.25 & 6 & 10.93 \\
\hline Avg & 1 & 0.22 & 4 & 10.88 & 5 & 11.10 \\
\hline Sep & 2 & 0.42 & 5 & 14.53 & 7 & 14.95 \\
\hline Oct & 2 & 0.92 & $=$ & - & 2 & 0.92 \\
\hline Nov & 3 & 20.35 & $\overline{1}$ & 12.00 & 4 & 32.35 \\
\hline Dec & 16 & 12.58 & 1 & 2.20 & 17 & 14.78 \\
\hline Total & 57 & 42.95 & 25 & 113.81 & 82 & 156.76 \\
\hline
\end{tabular}

The outage was classified as forced and scheduled. Hence, data on failure rates and repair times of component used in the generation system was compiled for reliability calculations. In addition, data on statistical information consisting of outages arising from the load shedding, system collapse, scheduled or unscheduled maintenance and hourly load shedding on each engine unit was collected. These data were used to compute the reliability indices, total hours of outages and the number of interruptions (frequency) per month.

Methods: In reliability analysis, the random variable is frequency time and so the standard function that best fit is the exponential function because it has only time as the independent variables. (Okah-Avae, 1995). Hence, the most important factor for this function that was used is that the hazard rate $(\lambda)$ which is the constant known as failure rate $(\lambda)$. (Roy et al., 1996) gave the density function as follows;

$\mathrm{F}(\mathrm{t})=\lambda e^{-\lambda t}$

And the hazard rate is given by $\lambda(\mathrm{t})=\frac{f(t)}{1-f(t)}$

where $(\lambda)=$ Failure Rate

$(\lambda)=\frac{\text { number of times that failures occured }}{\text { number of unit-hours of operation }}$

And the Reliability distribution function is given as:

$\mathrm{R}(\mathrm{t})=1-\mathrm{f}(\mathrm{t}) e^{\lambda t}$

Further Reliability parameter is given as follows: Mean Time between Failure (MTBF)

$M T D F=\frac{\text { Total System Operatin Hour }}{\text { Number Of Failures }}$

Mean Time To Repair (MTTR) or Mean Down-Time (MDT)

$$
\text { MTTR }=\frac{\text { Total Duration Of Outages }}{\text { Frequency of outages }}
$$

System Availability $(A)=\frac{\text { MTBF-MTTR }}{\text { MTBF }}$

Reliability Index $=\exp (-\mathrm{t} / \mathrm{MTBF})$

Maintainability Index $=1-\exp (-\mathrm{t} / \mathrm{MTTR})$

\section{RESULTS AND DISCUSSION}

Using equations 1-9, the reliability index, repair rate, failure rate, maintainability and availability index for generating stations 1 to 4 (G1 - G4) were computed with the results shown in Figure 1 to 5 . From the result presented, it was observed that G4 has the highest Failure rate of 0.0205 , followed by G1 (0.0156), GG2 (0.0141), and G3 (0.0115). Since G4 has the highest number of failures, then it is also expected to have the highest repair and maintenance rate.

This is confirmed in the result presented in Figure 2 and 3. The result revealed that $\mathrm{G} 4$ has the highest Repair Rate (0.7000), followed by G1 (0.5231), G2 (0.5140), G3 (0.4953) and the highest maintenance rate $(0.5032)$, followed by G1 (0.4073), followed by G2 (0.4019) and by G3 (0.3906). From Figure 4, it can be observed that G3 has the highest Availability (0.9767) per Unit, followed by G2 (0.9726) per Unit, G4 (0.9707) per Unit, and G1 (0.9701) per Unit. Since G3 has the highest availability, then it is also expected to have the reliability index. This was the case in the result shown in Figure 5. G3 is observed to have the highest Reliability (0.9880) per Unit i.e. 98.8\%, followed by G2 (0.9860) per Unit i.e. $98.6 \%$, G4 
(0.9854) per Unit i.e. $98.54 \%$, and G1 (0.9840) per unit i.e. $98.4 \%$.

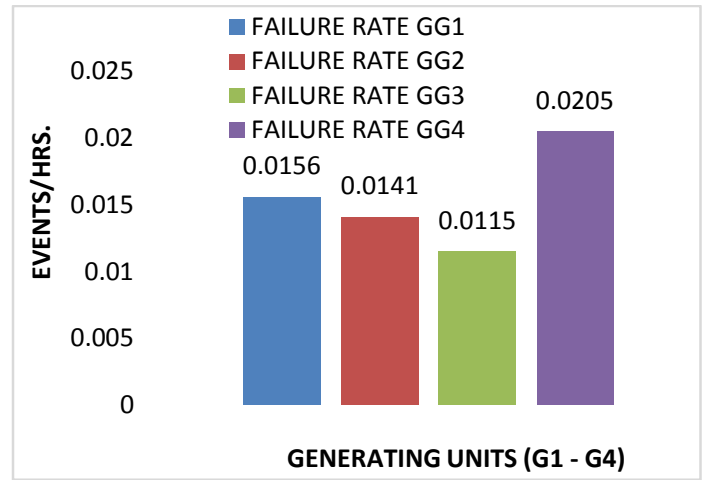

Fig 1: Comparative Failure Rate Curve

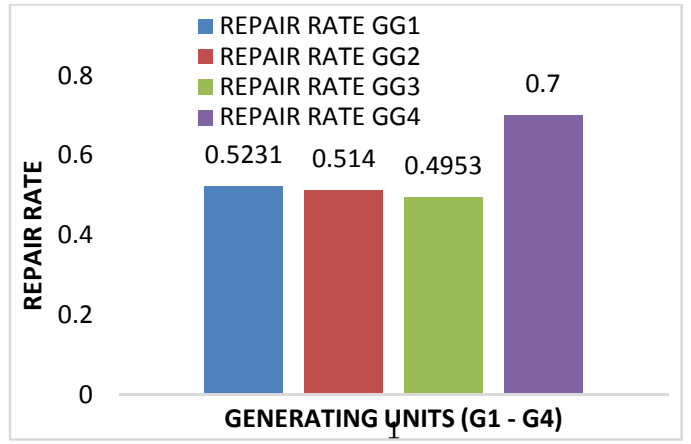

Fig 2: Comparative Repair Rate

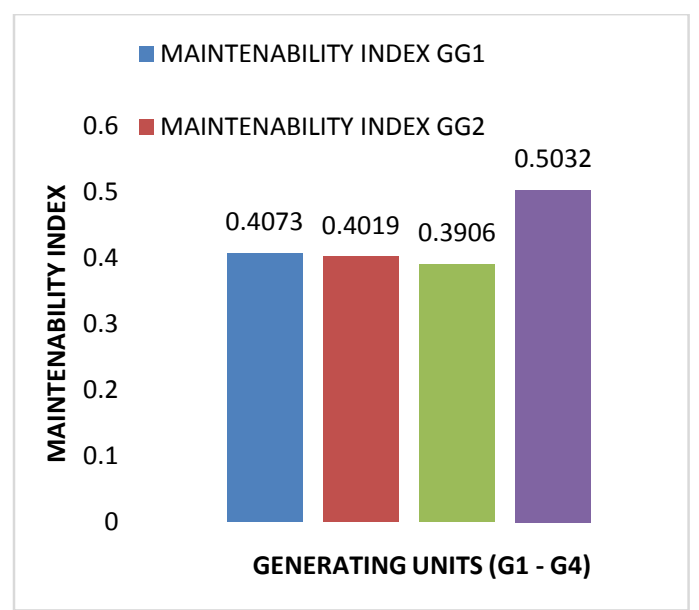

Fig 3: Comparative Maintainability Index

The result from this work implies that reliability and availability value for three generating unit were below the standard reliability values due to intermittent failures and several factors. Increasing failure rate is an intuitive concept caused by components wearing out and the causes of this failures are due to factors such as; weather conditions (storm, lighting, outdoor temperature and air humidity), contamination, Shortage of gas, human factor, excessive ambient temperature, moisture, excessive load, lack of maintenance, ageing, wear-out and design (circuit length, voltage system and circuit configuration). These factors make the component failure rates vary with time and location.

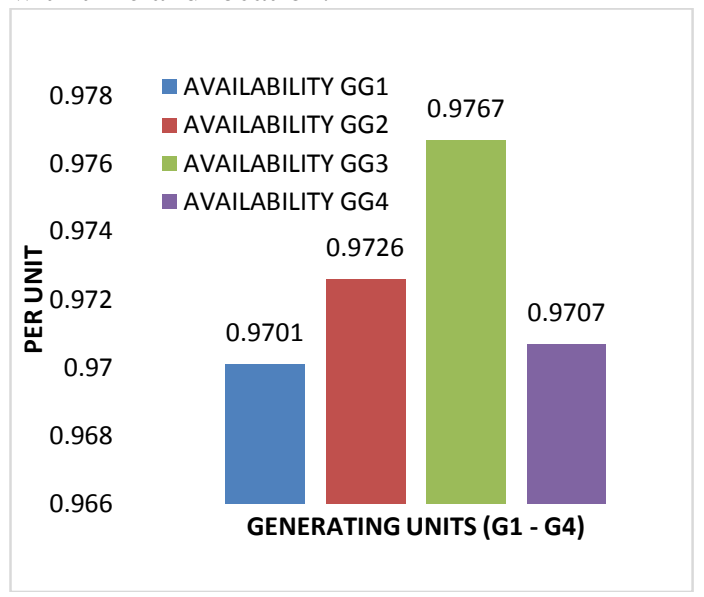

Fig 4: Comparative Availability Chart

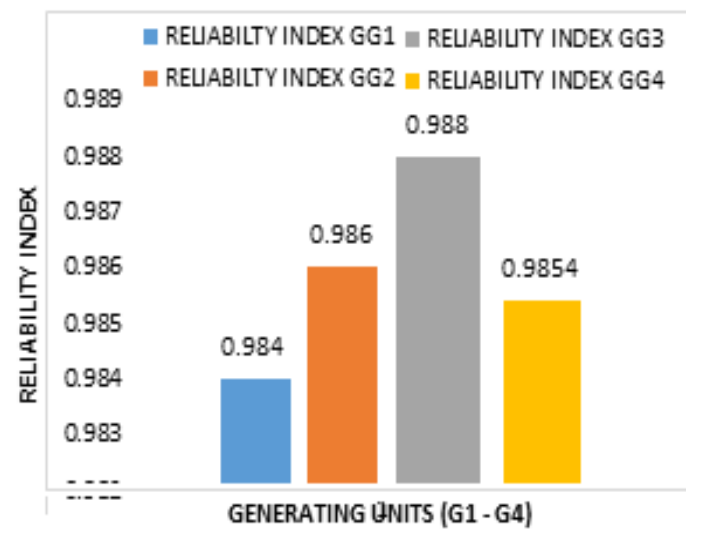

Fig 5: Comparative Reliability Index

Conclusion: This study has revealed that G3 is the most reliable generating unit in the plant and the only plant that meets the standard reliability requirement. Followed by G2, G4 and G1. Also, for the reliability and availability value of the generating stations to improve, the unit and capacity redundancy need to be incorporated into the design of the plant.

\section{REFERENCE}

Abdullah, M (2011). Essential aspect of power system planning in developing countries. Journal of King Saud University- Engineering Science, 23: 27-32.

Arya, R.; Choube, S; Arya, L (2012). Reliability evaluation and enhancement of distribution systems in the presence of distributed generation based on standby mode. International Journal of Electrical Power, 43(1): 607-616.

Billinton, R; Allan, RN (1988). Reliability assessment of a large Electric Power System. Boston. 
Deepak, S; Tri-Ratna, B; Mahesh, CL (2014). Reliability and Availability of Sunkoshi Hydro Power Plant. preceeding of IOE Graduate Conference.

Mangey, RA (2017). Gas Turbine Turbine Power Plant Performance Evaluation under key Failures. Journal of Engineering science and Technology, 12(7): 1871-1886.

Okah-Avae, B (1995). Science of Industrial Machinery and System Maintenance. Ibadan: Spectrum Books Limited Nigeria Limited.
Okwe, GI; Inyama, K (2013). Adequacy Analysis and Security Reliability Evaluation of Bulk Power System. IOSR Journal of Computer Engineering, 11(2): 26-35.

Roy, B; Allan, R; (1996). Reliability Evaluation of the Power System. Plenum Press

Sheng Z; Tianmeng Y; Yaowu W; Suhua L; Taijun L (2017). The Reliabilty Evaluation Method of Generation System Based on the Importance Sampling Method and States Clustering. Energy Procedia. 118: 128-135. 